\title{
Recherches sur le Développement post-embryonnaire de la Langouste commune (Palinurus vulgaris).
}

\author{
Par \\ M. E.-L. Bouvier, \\ Professeur au Mluséum d'Histoire Naturelle à Paris. \\ Avec 6 Figures dans le Texte.
}

C'EsT surtout pour étudier le développement post-embryonnaire de la Langouste commune (Palinurus vulgaris Latr.) que je me suis rendu au Laboratoire de Plymouth. L'endroit me semblait excellent parce que la Langouste n'est pas rare près des côtes dans les eaux du Devonshire et de la Cornouaille, parce qu'elle est principalement fréquente sur les fonds rocheux au-dessus desquels se dresse le phare d'Eddystone, aussi enfin, parce que le Laboratoire de Plymouth est très bien installé, avec un bateau, l'Oithona, qui peut se rendre en mer chaque jour et effectuer les pêches les plus diverses.

Car il fallait pêcher souvent et à toutes profondeurs pour atteindre le but que je m'étais fixé, et ce but n'était rien moins que de découvrir les stades jusqu'alors inconnus du développement de notre Langouste. Depuis les observations de Couch $(1857,25)$ justifiées par Gerbe $(1858$, 547 ) et par Dohrn (1870), mais à tort contestées par Sp. Bate (1868), on sait que le $P$. vulgaris sort de l'œuf sous une forme larvaire foliacée, hyaline, aplatie dans le sens dorso-ventral, que les anciens zoologistes avaient appelée phyllosome et tenaient pour un genre spécial de Crustacés décapodes. On savait aussi, depuis les recherches de Claus (1863), que le phyllosome acquiert progressivement des appendices à mesure qu'il se développe, qu'il peut atteindre une longueur de $21 \mathrm{~mm}$., et qu'il garde jusque dans sa plus grande taille les caractères généraux qui lui sont propres et qui en font un organisme essentiellement pélagique. Mais quelle est la série des stades présentés par le phyllosome depuis la sortie de l'œuf jusqu'au moment où il acquiert la forme longue, trapue, et rétrécie des Decapodes normaux? et comment s'effectue le passage à cette forme? Autant de questions qu'il fallait se poser et que personne encore n'avait pu résoudre. A vrai dire, depuis 
les belles recherches de M. Boas (1881) récemment confirmées par M. Calman (1909), on était en droit de croire que le passage du phyllosome à la forme définitive s'effectue par le moyen d'un "stade natant" désigné jadis par M. Ortmann (1897) sous le nom de puerulus et considéré par cet auteur comme un genre autonome de Palinurien. J'ai moi-même apporté ma contribution (1912) aux travaux de ces auteurs et je connais actuellement le puerulus supposé d'une dizaine d'espèces de Langoustes. Mais ces Langoustes sont toutes exotiques, et d'ailleurs, c'est par une hypothèse rationnelle, mais non à la suite d'une observation directe, que l'on s'accorde à voir dans le puerulus le stade intermédiaire qui conduit du phyllosome à l'état de Langouste parfaite. Il convenait par suite de justifier les suppositions de M. Boas et de chercher à connaître tous les stades évolutifs ainsi que le puerulus de l'espèce localisée dans nos mers. Il fallait aussi chercher l'habitat des puerulus; car ces organismes, au contraire des phyllosomes, sont d'une rareté extrême, et c'est tout au plus si l'on en possède une trentaine d'exemplaires, pour la totalité du groupe des Palinuriers.

La saison d'été m'a paru plus propre que toute autre à la solution de ces problèmes, car c'est dans la seconde quinzaine de juin et au début de juillet que M. Cunningham (1891-92) captura autour du phare d'Eddystone les premiers stades larvaires du $P$. vulgaris. Mon savant prédécesseur a très bien décrit et figuré ces stades, mais, peu favorisé par le temps, il n'a pu en obtenir que deux, et les pêches faites dans la suite ont été infructueuses, alors qu'elles auraient dû lui procurer la capture des stades plus âgés. C'est dans l'espoir d'obtenir ces stades que je me suis installé à Plymouth du 15 juillet au $1^{\mathrm{er}}$ septembre. Le bref exposé qu'on va lire montrera que le moment était bien choisi; j'ajouterai, d'ailleurs, que je fus étrangement favorisé par une saison des plus propices.

\section{Stades phyllosomes de la Langouste commune.}

Je vais indiquer tout d'abord les stades phyllosomes obtenus par l'Oithona au cours decet te saison de pêches. L'étude n'en est pas. encore achevée et c'est provisoirement que je les classe en série de la. manière suivante :

Stade 1 (3 mm. environ). Antennules et antennes presque indivises, de longueur à peu près égale. Le bouclier céphalique atteint à peine la base des maxillipèdes postérieurs ( $m x p .3)$; exopodite des péréiopodes 3 ( $p .3)$ dépourvu de soies, péréiopodes des deux paires suivantes ( $p .4$, $p$. 5) à l'état de bourgeons très courts. Abdomen sans appendices visibles et sans articulations bien distinctes en dehors du telson. Ce. 
stade a été parfaitement figuré par M. Cunningham dans la première figure de son mémoire; il est celui que présente le phyllosome à sa sortie de l'œuf.

Stade 2 (4 à $5 \mathrm{~mm}$.). Un court bourgeon représente le fouet interne des antennules; $p .5$ et surtout $p .4$ plus allongés qu'au stade précédent; des uropodes légèrement échancrés au bout et atteignant au plus le milieu du telson, qui est tronqué en arrière; la segmentation de l'abdomen encore très vague.

Stade 3 (6 à $8 \mathrm{~mm}$.). La portion pédonculaire des antennules est divisée en deux articles, le fouet interne égale environ un tiers du fouet externe. Le pédoncule des antennes est indivise, et un peu plus court que le fouet, qui est également indivise. Le céphalon recouvre la base de mxp. 3; mxp. 2 avec un bourgeon exopodial. Des soies à l'exopodite de $p .3, p .4$ avec un exopodite nu. Des pléopodes representés par un bourgeon aplati et échancré en arrière; uropodes plus longs qu'au stade 2 et profondément échancrés en deux rames; segmentation de l'abdomen bien distincte, surtout dans les régions épimérales. C'est le dernier stade observé par M. Cunningham, qui l'a bien décrit et fort exactement figuré.

Stade $4(9 \mathrm{~mm}$.). Le pédoncule antennulaire commence à montrer une division en 3 articles. Il y a déjà quelques soies sur l'exopodite de $p .4$; cette patte à peu près aussi longue que l'abdomen. Les pléopodes sont nettement biramés; le telson est arrondi sur son bout distal et légèrement plus long que les uropodes dont l'exopodite présente une très nette échancrure externe.

Stade $5(10 \mathrm{~mm}$.). Le pédoncule antennulaire est nettement divisé en 3 articles; les antennes sont un peu plus longues que les antennules. L'exopodite de $p .4$ atteint presque le bout de l'endopodite ou, tout au moins, en dépasse le milieu.

Stade 6 (12-13 mm.). Le fouet interne des antennules égale à peu près la moitié du fouet externe; le fouet antennaire égale une fois et demie la longueur de son pédoncule. L'exopodite de mxp. 2 atteint l'extrémité distale de l'article suivant; $p .5$ arrive à peine à la base des uropodes; les soies de p. 4 bien développées.

Stade 7 (14-15 mm.). Le fouet antennaire égale près de deux fois la longueur du pédoncule; $m x p .1$ est déjà long; l'exopodite de $m x p .2$ dépasse l'extrémité distale de l'article suivant. Ce stade a été assez bien figuré par Claus (1863, Taf. XXVI, fig. 7) et avec quelques erreurs par J. Couch (1858).

Stade 8. N'a pas encore été trouvé. 
Stade 9 (20-21 mm.). (Fig. 1.) Le fouet interne des antennules égale presque le fouet externe ; les antennes sont à peu près aussi longues que le céphalo-thorax, et leur fouet, où l'on voit déjà des traces de segmentation, égale environ trois fois la longueur du pédoncule. L'exopoditedes mâchoires est bien développé. Exopodite de mxp. 1 long et formé de 2 articles, toutes les autres parties de l'appendice (sauf probablement l'épipodite) sont bien indiquées mais réduites; exopodite de $m x p .2$ à peu près aussi long que l'endopodite, mais dépourvu de soies. Appareil branchial formé des mêmes parties que celui de l'adulte, mais les épipodites de mxp. 1 et mxp. 2 font probablement défaut; le céphalon recouvre la

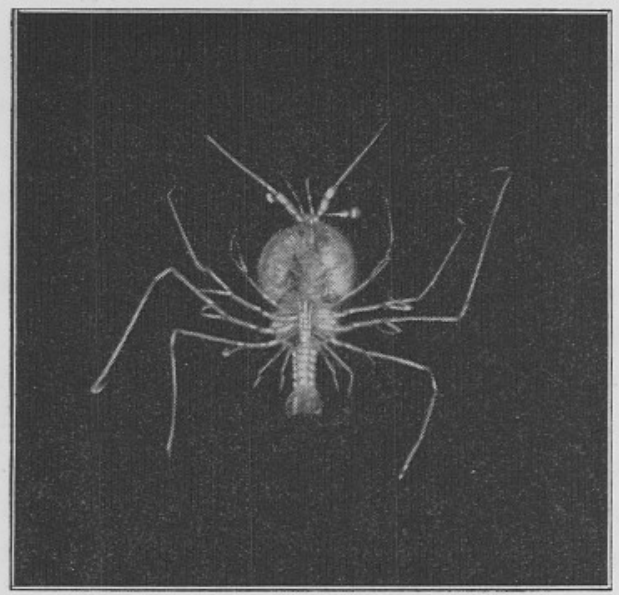

Fig. 1.-Phyllosome de $P$. vulgaris à l'avant-dernier stade (stade 9) capturé près du phare d'Eddystone. Photographié au Laboratoire d'entomologie du Muséum par M. Boyer. (Cliché communiqué par "Science et Vie".) Grandeur naturelle.

base de $p$. 1. Les épimères abdominaux sont toujours arrondis, les pléopodes ont un pédoncule très net. Les pédoncules oculaires, grâce à leur tigelle fort longue, atteignent le bout distal des antennules et dépassent le pédoncule des antennes. C'est le dernier stade observé jusqu'ici; Claus en a donné la description et la figure (1863, Taf. XXVI, fig. 8); il a été passablement représenté par Spence Bate (1863, fig. 1).

Stade 10 (20-21 mm.). Ce stade très intéressant était resté inconnu, non seulement dans notre Langouste commune, mais chez tous les Palinuridés. On en trouvera ci-contre la figure (fig. 2). Ce qui le distingue essentiellement, c'est la structure de l'abdomen qui se rapproche déjà beaucoup de celle de la Langouste adulte; car le telson présente deux 


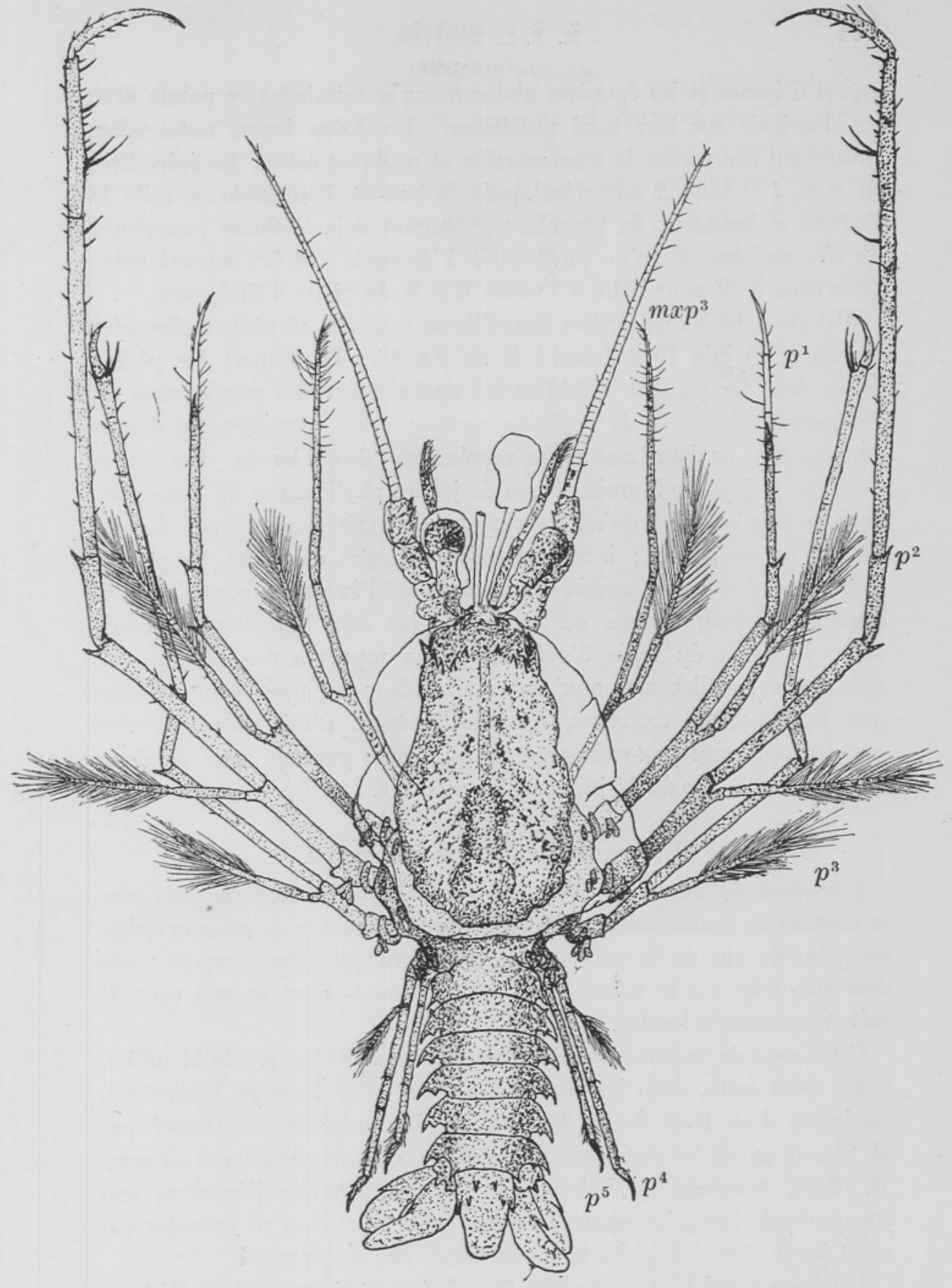

Fig. 2.-Phyllosome de $P$. vulgaris an dernier stade (stade 10) et montrant le puerulus qui commence à s'en dégager. Sous la carapace un peu déformée du phyllosome se voit le bouclier céphalo-thoracique du puerulus, un pédoneule oculaire de ce dernier est sorti du pédoncule phyllosomien, l'autre est encore coiffé de son exuvie; certaines pattes commencent également à se dégager. Dessin de l'auteur d'après un exemplaire capturé dans les parages d'Eddystone. (Le cliché a été communiqué par "Science et Vie".) Gross. 4. 
paires d'épines et les épimères abdominaux se terminent en pointe avec un denticule sur leur bord postérieur. Les longs fouets antennaires présentent des traces de segmentation et quelques soies; les épipodites de $m x p$. 1 et $m x p$. 2 sont développés, la formule branchiale est celle de l'adulte et beaucoup de branchies présentent déjà quelques bourgeons. Je n'ai pu obtenir qu'un phyllosome à ce stade; il fut capturé entre deux eaux le 25 août 1913, à 5 milles E.N.E. du phare d'Eddystone.

J'ai examiné les matériaux recueillis au cours de 46 pêches effectuées depuis le 20 juin 1913, jusqu'à la fin d'août. La plupart des pêches faites dans les parages d'Eddystone renfermaient des phyllosomes de Palinurus vulgaris et de Scyllarus arctus; celles pratiquées en dehors de cette zône ne donnèrent aucun représentant de ces larves. Les pêches du mois de juin ne fournirent que de jeunes phyllosomes de Palinurus, mais je dois observer qu'au Laboratoire on avait conservé peu de matériaux de ces pêches; à mesure qu'on avançait en juillet, les phyllosomes âgés devenaient nombreux et sûrement l'évolution peut s'achever durant ce mois, comme on le verra plus loin. D'ailleurs, durant toute la durée du mois d'août, la pêcha rapporta des phyllosomes âgés et des phyllosomes aux premiers stades; on peut donc affirmer que la Langouste commune, dans les parages d'Eddystone, effectue son développement post-embryonnaire au moins pendant toute la durée de l'été, c'est-à-dire du 21 juin au 21 septembre.

\section{Stade Natant ou Puerulus.}

Caractères du puerulus. Pour des raisons que j'indiquerai plus loin, la capture de l'exemplaire précédent a une importance de premier ordre, au point de vue de la solution des problèmes qui nous occupent; elle doit être mise sur le même rang qu'une seconde dont je vais tout de suite entretenir le lecteur.

Cette seconde capture est celle d'un puerulus qui fut pris le 31 juillet, entre deux eaux, dans les parages compris entre Looe et Eddystone, au-dessus d'un fond de 27-29 brasses. L'exemplaire fut trouvé par M. Gossen parmi les matériaux recueillis au cours de pêches que dirigeait M. Clark, Assistant du Laboratoire. Faut-il dire que j'éprouvai une joie profonde lorsqu'on apporta sur ma table de travail ce puerulus qui avait jusqu'alors échappé à la connaissance des zoologistes ?

L'unique et précieux exemplaire (figs. 3, 4, 5, 6) mesure environ $21 \mathrm{~mm}$. de longueur, ce qui est la taille des grands phyllosomes. Comme tous les puerulus, il est hyalin, avec des téguments coriaces à peu près totalement dépourvus de calcification, et la forme macrourienne normale. Il se 
distingue pourtant des puerulus actuellement connus par son bouclier céphalo-thoracique regulièrement convexe, mais il est possible que cette disposition soit due au renflement des régions branchiales sous l'action de l'eau de mer où l'animal a péri, ou des liquides conservateurs; dans les autres puerulus, en effet, la carapace est très peu convexe dorsalement, et ses régions branchiales forment un angle presque droit avec la région dorsale, disposition qui s'est conservée chez un certain nombre de Palinuridés primitifs (Puerulus Ortm.; Linuץarus Gray). D'ailleurs, notre puerulus est bien au "stade natant" décrit par M. Boas, car ses

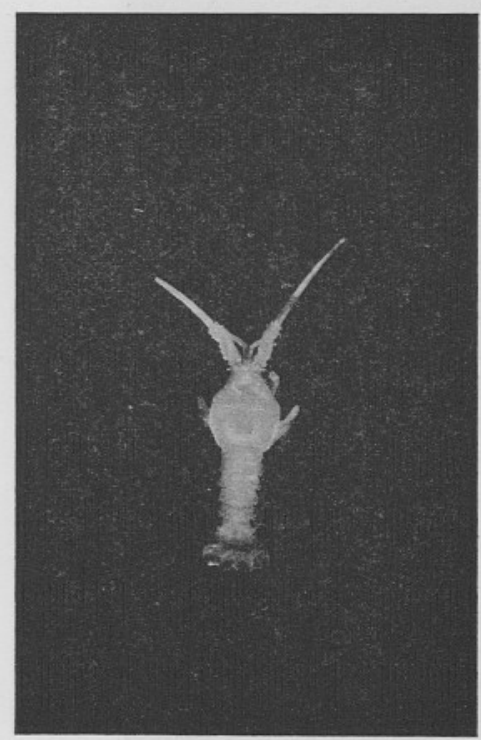

Fig. 3.

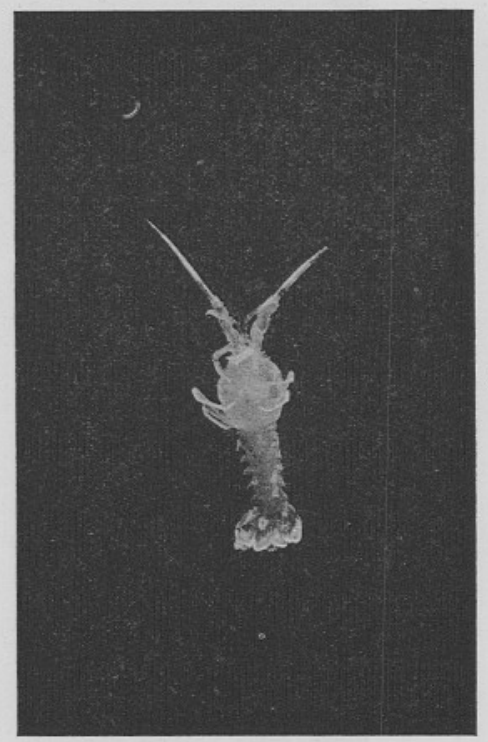

Fig. 4.

FIG. 3.-Puerulus de P. vulgaris capturé dans les parages d'Eddystone. Face dorsale (d'après une photographie de M. Martin Duncan prise au Laboratoire de Plymouth). Grandeur naturelle.

Fra. 4.-Puerulus de la Fig. 3, vu du côté ventral (d'après une photographie de M. Martin Duncan).

pléopodes peuvent se conjuguer par couples dans une même paire, grâce aux rétinacles à crochets qui occupent le bout distal de l'appendice interne situé sur leur rame endopodiale.

L'exemplaire capturé présente déjà de grandes resemblances avec la forme définitive du Palinurus vulgaris; les antennules sont du même type brévicorne, c'est-à-dire terminées par deux courts fouets subégaux dont l'externe est beaucoup plus épaissi que l'autre;-les antennes sont très fortes, avec un long et puissant fouet bien articulé et un large 


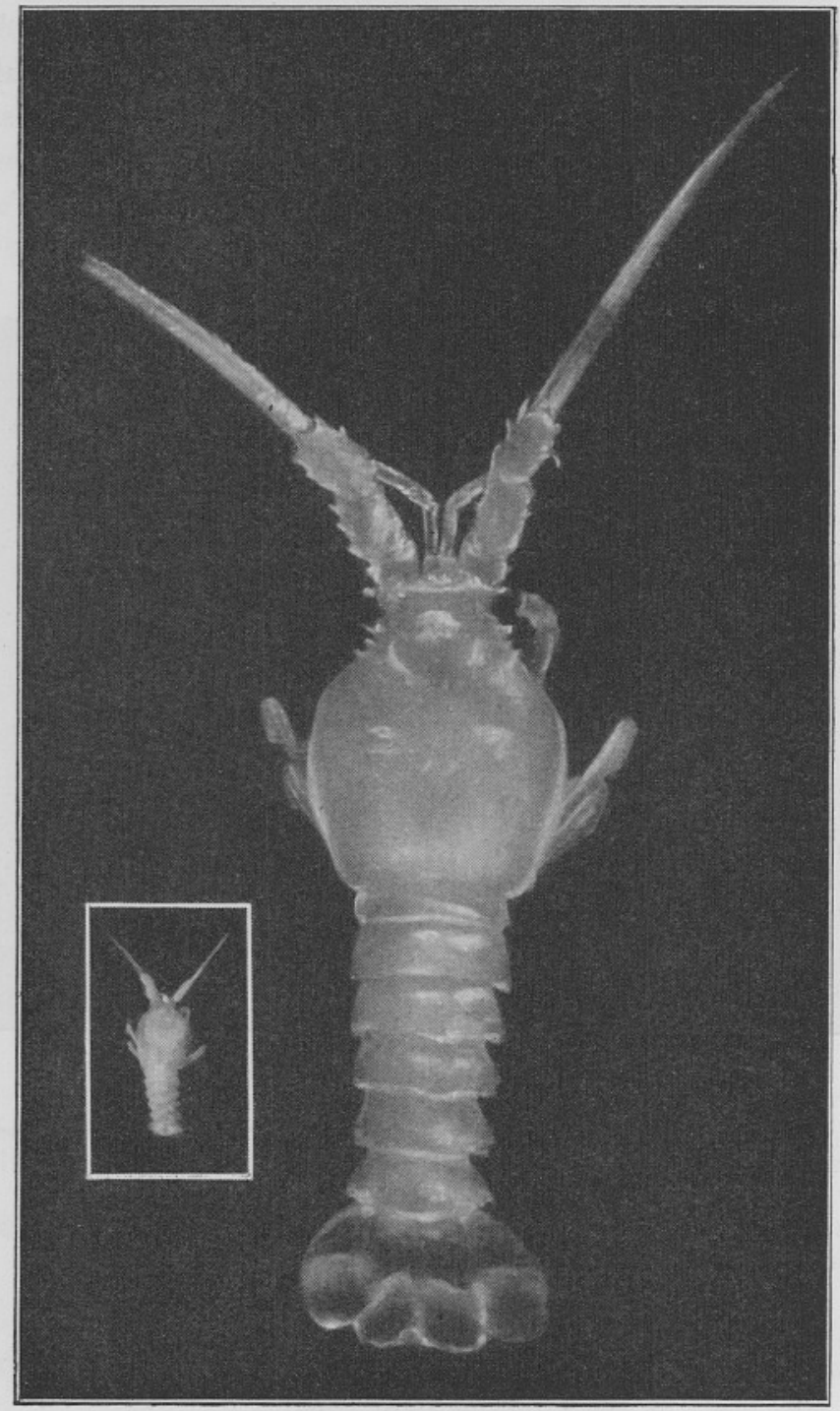

Fig. 5. - Puerulus des denx figures précédentes vu du côté dorsal. (Agrandissement photographique obtenu par M. Martin Dunean.) 
pédoncule où les épines sont distribuées à très peu près comme chez la Langouste adulte;-les pédoncules oculaires sont courts et larges;le rostre est réduit à une faible pointe médiane comme dans les espèces du genre Palinurus; - -les cornes rostrales sont très puissantes et suivies d'une paire d'épines post-rostrales;-il y a sur la carapace quelques épines qu'on retrouve chez l'adulte (une rangée transverse de quatre épines gastriques, une paire de spinules cardiaques, et, au bord supérieur de chaque région branchiale, une dizaine de spinules disposées en série)les épimères abdominaux sont aigus et, sauf le premier, munis d'une saillie en pointe sur leur bord postérieur; - on observe une paire de fortes pointes sur le $6^{\mathrm{e}}$ segment abdominal, à la naissance du telson ;ce dernier présente déjà la paire d'épines proximales et les échancrures latérales épineuses de la Langouste adulte;-les uropodes sont munis, comme dans cette dernière, d'échancrures externes spinuleuses, les pattes enfin sont courtes, fortes, et dépourvues d'exopodites* bien développés.

Il y a pourtant de profondes différences entre ce puerulus et la forme définitive de la Langouste commune; outre les caractères généraux propres à tous les puerulus (corps hyalin, téguments coriaces et non calcifiés; présence de rétinacles sur les appendices internes des pléopodes) il faut signaler surtout l'absence de denticulations aiguës sur les cornes rostrales (fig. 6), le développement de soies assez longues sur les fouets antennaires, l'absence de sillons transverses sur les segments abdominaux et surtout l'armature épineuse de la carapace qui est loin d'être riche et puissante comme dans la Langouste adulte. J'ai montré plus haut que les épines du puerulus se retrouvent à leur place dans la forme définitive; cela est vrai pour toutes, sauf peut-être pour trois épines, probablement hépatiques, situées de chaque côté en arrière des antennes ; ces épines sont fortes et très caractéristiques de notre puerulus, mais il est difficile de leur trouver des homologues dans les nombreuses épines qui ornent le bouclier céphalo-thoracique de l'adulte.

Le puerulus d'Eddystone se rapproche surtout d'un puerulus caraïbe que j'ai rapporté $(1912,81)$ au Palinurus longimanus Edw.; il présente comme lui un exopodite flagellé sur les maxillipèdes externes et plusieurs paires d'épines sternales; ces dernières, toutefois, sont plus développées dans notre puerulus et d'ailleurs plus nombreuses, car on en trouve une à la base des pattes des quatre paires.postérieures tandis que les épines se localisent à la base des trois dernières paires de pattes dans le puerulus caraïbe.

* L'exemplaire avait perdu quelques-unes de ses pattes, mais celles qui restent sont courtes et fortes comme dans les puerulus des autres Palinuridés. 


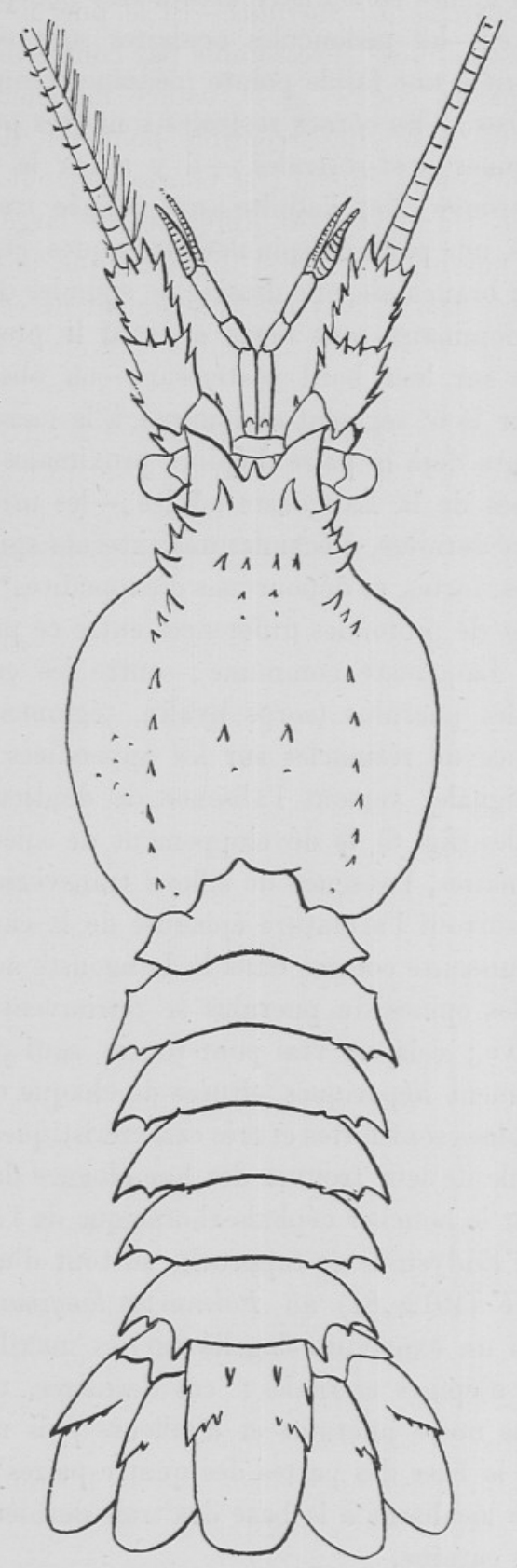

Fig. 6.-Esquisse du même puerulus montrant les ornements en saillie de la face dorsal. Dessin de l'auteur d'après l'exemplaire capturé dans les parages d'Eddystone. (Cliché communiqué par "Science et Vie".) 
Passage du phyllosome au puerulus.-Si le puerulus ressemble beaucoup à la Langouste adulte, il ressemble par contre bien peu au phyllosome: avec ses pattes courtes de Crustacé marcheur, ses exopodites thoraciques réduites à un imperceptible bourgeon dépourvu de soies, ses pédoncules oculaires à tigelle très courte, surtout avec son bouclier céphalo-thoracique trapu et très différent du large et plat bouclier phyllosomien, le puerulus est aussi différent que possible de la forme larvaire des Palinuridés. Provient-il réellement de cette forme et comment s'effectue le passage de l'un à l'autre ? Voilà ce qu'il fallait encore trouver.

Par une bonne fortune singulière et qu'il eût été fou d'espérer, l'Oithona, sous la direction de M. Clark, fît capture d'un phyllosome dans lequel était en voie de se former et de se dégager le puerulus. Ce phyllosome est celui (fig. 2) dont j'ai donné plus haut la description et qui représente, à mon avis, le $10^{\mathrm{e}}$ stade larvaire de notre Langouste, un stade que les zoologistes n'avaient pas encore observé. Son abdomen ressemble déjà tout à fait à celui du puerulus, mais le spécimen est bien phyllosome par tous ses autres caractères.

Ce phyllosome fut rapporté vivant par M. Clark qui me prévint de suite; mais le délicate organisme avait succombé aux fatigues du voyage et était complétement inerte quand j'arrivai au laboratoire pour l'examiner. La nuit était venue, on le conserva dans l'alcool et j'en fis l'étude le jour suivant. Sa carapace était déformée et ses pédoncules oculaires avaient un aspect bizarre, si bien qu'il me parut être tout d'abord un phyllosome en mauvais état. Mais un examen plus attentif modifia bien vite cette conception trop rapide: l'abdomen avait des caractères tout particuliers propres au puerulus, et sous le bouclier céphalo-thoracique déformé, on apercevait, comme une masse opaque, le bouclier même du puerulus, avec son rostre minuscule, ses cornes frontales, ses fortes épines hépatiques et la plupart des spinules de la carapace (fig. 2). Le pédoncule oculaire droit du puerulus était déjà sorti du pédoncule phyllosomien; le pédoncule oculaire gauche était également formé, indépendant de la tigelle phyllosomienne, pourtant coiffé encore du pédoncule phyllosomien; certaines pattes du puerulus commençaient à se dégager des pattes phyllosomiennes.

En fait, c'était une pièce merveilleuse et singulièrement démonstrative que permettait d'établir sans contestation possible : $1^{\circ}$ que le phyllosome capturé représente bien le dernier stade larvaire de notre Langouste; $2^{\circ}$ que cette dernière ne traverse pas, comme d'autres Palinuridés, le curieux stade PHYLLAMPHION décrit et figuré par Reinhardt en $1858 ; 3^{\circ}$ que les con 
ceptions de M. Boas sont parfaitement justes en ce sens que le puerulus est bien directement issu du phyllosome, du moins dans notre Langouste commune.

Par analogie, on devra étendre cette dernière et très importante conclusion à tous les Palinuridés, en remarquant toutefois que certaines formes exotiques traversent un stade phyllamphion, assez peu différent d'ailleurs du phyllosome.

Passage du puerulus à la Langouste.-Les observations précédentes nous donnent-elles le droit de conclure que le puerulus d'Eddystone est celui de la Langouste commune et, d'une manière plus générale, que les divers puerulus connus représentent le "stade natant" de divers Palinuridés? Cela ne me paraît point douteux. Le Palinurus vulgaris représente à lui seul, dans nos mers, la famille des Palinuridés, d'où cette conclusion que tous les phyllosomes palinuriens capturés à Eddystone proviennent bien de cette Langouste. Nous en dirons autant du puerulus, car nous avons vu ce dernier prendre naissance au sein même des grands phyllosomes. Le puerulus d'Eddystone est done, sans incertitude aucune, le "stade natant" du Palinurus vulgaris.

Il s'en faut que l'on puisse conclure, avec une précision semblable, lorsqu'on s'adresse aux autres formes de la famille, car on n'a jamais observé, dans ces dernières, la série complète des stades phyllosomes et moins encore le passage du phyllosome au puerulus. Mais j'ai largement établi, dans un travail antérieur (1912), que chaque puerulus présente des caractères qui permettent de le rapporter, presque sûrement, à une espèce spéciale de Palinuridés.

\section{Habitudes des Phyllosomes et des Puerulus.}

Toutefois les suppositions les plus logiques ne valent pas une bonne preuve et, en ce qui concerne la Langouste commune, il faudra observer le passage du puerulus à la forme définitive. Ce sera l'objet principal de mes recherches durant la campagne que je dois effectuer prochainement au laboratoire de Plymouth.

La principale difficulté sera d'obtenir des puerulus. On peut se les procurer par deux moyens : soit en élevant des phyllosomes de grande taille, soit en les capturant directement au large.

La première méthode sera sans doute la meilleure et la plus sûre, car l'élevage en aquarium des grands phyllosomes ne semble pas devoir être très difficile. M. Clark a capturé, dans les parages d'Eddystone, un phyllosome à l'avant-dernier stade et l'a rapporté vivant au laboratoire où j'ai pu l'observer pendant les deux jours qui précédèrent mon 
départ. Quoique placé dans une simple jarre et dans des conditions peu favorables, la curieuse larve paraissait très active et en fort bon état; elle se déplaçait à peu près exclusivement au moyen de ses exopodites thoraciques, qui lui servaient aussi à se maintenir dans le milieu et à la diriger ; elle descendait lentement quand ces appendices n'étaient plus en fonction; les pattes elles-mêmes font peu de mouvements et semblent jouer le rôle de balanciers, l'abdomen et ses pléopodes n'en font aucun. Le jeune animal est rapidement et fortement attiré par la lumière. A cet avant-dernier stade, les phyllosomes sont assez communs vers la fin de juillet et durant le mois d'août; en les élevant dans un aquarium convenable, on pourra peut-être les conduire jusqu'au dernier stade et de celui-ci au "stade natant" ou puerulus.

La capture des puerulus en pleine mer sera plus difficile. Ces jeunes, en effet, ne doivent pas rester longtemps entre deux eaux, dans le milieu où ils se trouvent en sortant des phyllosomes, et où fut capturé l'exemplaire que j'ai décrit plus haut. Ce sont des nageurs au corps lourd, qui doivent très vite descendre sur le fond* où ils se cachent certainement parmi les rochers, car leurs téguments coriaces et non calcifiés les rendent très vulnérables. Ainsi doit s'expliquer, à mon avis, l'extrême rareté des puerulus dans les collections zoologiques et la diversité d'habitat jusqu'ici connue chez ces animaux: les puerulus étudiés par M. Boas avaient été, "en partie du moins, capturés au large" $(1880,84)$, et je sais, d'après les notes des pêches faites par la Princesse Alice, que le type du puerulus atlanticus ("stade natant" du Palinurus regius $\mathrm{Br}$. Cap.) fut capturé au trémail par 20 mètres de profondeur, à Ste. Lucie du Cap Vert. D'un autre côté, M. Calman rapporte que le puerulus spiniger, Ortm. ("stade natant" du Palinurus ornatus Fab.) fut trouvé à l'île Christmas "soit au milieu des rochers, soit sur le "pier" dans les crevasses des piles" $(1909,444)$, et j'ai noté ailleurs $(1912,88)$ que le puerulus du Panulirus dasypus Latr. fut trouvé par M. le Dr. Jousseaume dans les crevasses du rivage, à Djiboutil.

Qu'ils soient obtenus par élevage ou par capture directe, les puerulus, à cause de leurs habitudes, se prêteront certainement beaucoup mieux que les phyllosomes à la captivité de l'aquarium. On pourra, sans difficulté, j'en suis sûr, les conduire jusqu'à la forme définitive de l'espèce à laquelle ils appartiennent. Cette forme sera sans doute acquise à la première mue, car les puerulus d'une espèce ont tous à peu près la même

* Il en est, sans doute, de même pour les Homards ; le bateau du laboratoire a capturé, entre deux eaux, un jeune Homard qui avait acquis tous les caractères definitifs de l'espèce; le jeune venait sûrement de sortir de sa mysis qui est pélagique. 
taille, et cette taille n'est pas moins grande que celle des plus jeunes Langoustes. En capturant à Djiboutil les puerulus du Panulirus dasypus, M. Jousseaume a recueilli plusieurs jeunes de l'espèce, et ces jeunes présentent la même taille et les mêmes variations de taille que les puerulus;* le Muséum d'histoire naturelle possède un jeune exemplaire de la Langouste commune qui dépasse de $4 \mathrm{~mm}$. seulement le puerulus d'Eddystone, et l'on trouverait sûrement des jeunes ayant la même taille que ce dernier. Quoiqu'il en soit, il n'est pas douteux que du puerulus sortira la Langouste sous sa forme définitive.

J'arrête là cette note préliminaire qui sera complétée et détaillée à la suite de mon prochain séjour au Laboratoire de Plymouth. Si, comme je l'espère, on lui trouve quelque intérêt, je dois en remercier toutes les personnes qui ont provoqué ou facilité mes recherches, c'est-à-dire les membres de la "Marine Biological Association," M. le Professeur Ray Lankester qui me désigna comme "investigator" au choix de cette Société, et, pour leur dévouement au-dessus de tout éloge, M. Allen, Directeur du Laboratoire de Plymouth, M. Clark, assistant, M. M. Gossen et Savage attachés au service, et sans aucune exception le personnel du Laboratoire. Je dois aussi remercier mon excellent confrère, M. F. Martin Duncan, qui travaillait au Laboratoire durant mon séjour à Plymouth et qui a très obligeamment photographié, peu après sa capture, le précieux puerulus recueilli par l'Oithona.

\section{IINDEX BIBLIOGRAPHIQUE.}

1868. Bate, C. Spence. Carcinological Gleanings. No. IV. Ann. and Mag. Nat. Hist. (4). Vol. II, pp. 116, 117, Pl. X!figs. 1 et 2.

1880. Boas, J. E. V. Studier over Decapodernes Slaegtskabsforhold. Vid. Selsk. Skr. X, 6 R., nat. og mat. Afd. 1 et 2, pp. 78-85.

1912. Bouvier, E. L. Le stade " natant" ou "puerulus" des Palinuridés. Trans. of the Second Entomological Congress, pp. 78-89 (avec la bibliographie complète relative aux puerulus).

1913a. Id. The Post-Embryonic Development of the Spiny Lobster. Nature. Vol. 91, pp. 633, 634 (une figure dans le texte).

1913b. Id. Observations nouvelles sur le développement larvaire de la Langouste commune (Palinurus vulgaris Latr.). Comptes rendus Ac. des Sciences. T. 140, pp. 457-463 (avec une figure dans le texte).

* Les puerulus capturés par M. le Dr. Jousseaume mesurent de 15 a $19 \mathrm{~mm}$. et les jeunes Langoustes 18 à 20 . 
1913c. Id. La Langouste royale. La Science et la Vie. No. 9, pp. 310-320. (avec figures dans le texte).

1909. Calman, W. T. The genus Puerulus Ortmann and the Post-larval Development of the Spiny Lobsters (Palinuridae). Ann. and Mag. Nat. Hist. (8), Vol. 8, pp. 441-446.

1863. Claus, C. Ueber einige Schizopoden und niedere Malakostraken Messina's. Zeit. Wiss. Zool., B. XIII, pp. 422-433. Taf. XXV, XXVI.

1858. Couch, J. Note on the Occurrence of Phyllosoma commune on the Coast of Cornwall. Journ. of the Proc. Linn. Soc., Zool., Vol. II, pp. 146-149 (avec figures dans le texte).

1857. Couch, R. Q. On the Embryo State of Palinurus vulgaris. Rep. Brit. Assoc. Advanc. of Science, 1857, pp. 102-103.

1858. Id. On the Embryo State of Palinurus vulgaris. Nat. Hist. Review, Vol. IV, pp. 250-251. Pl. XVIII.

1892. Cunningham, J. T. On the Development of Palinurus vulgaris, the Rock Lobster or Sea-Crayfish. Journ. Mar. Biol. Association, Vol. II. (N.S.), pp. 141-150, Plates VIII et IX. 1891-92 (excellent exposé bibliographique relatif aux phyllosomes).

1870. Doнrn, A. Untersuchungen über Bau und Entwicklung der Arthropoden. VI. Zur Entwicklungsgeschichte der Panzerkrebse, Decapoda loricata. Zeit. Wiss. Zool., B. XX, pp. 248-271, Taf. XVI.

1858. Gerbe, Z., in COSTE. Note sur la larve des Langoustes. Comptes rendus Ac. des Sciences. T. 46, pp. 547-548, et Rev. et Mag. de Zool. (2). T. X, pp. 134-136.

1858. Gú́rin-Méneville, F. E. Phyllosome ... premier état des Langoustes. Soc. ent. de France (3). T. VI. Bulletin, pp. xlivxlv.

1897. Ortmann, A. E. On a New Species of the Palinurid genus Linuparus, found in the Upper Cretaceous of Dakota. Am. Journ. of Science. Vol. IV, p. 290 (note).

1850. Reinhardt, F. Phyllamphion en ny Slaegt af Stomatopodernes Orden. Vid. Medd. Naturh. For. 1849-1850, pp. 2-6, et 1858, Tab. I (A).

1873. Richters, Ferd. Die Phyllosomen, ein Beitrag zur Entwicklungsgeschichte der Loricaten. Zeit. Wiss. Zool., B. XXIII, pp. 621646, Taf. XXXI-XXXIV. 